\title{
A Low Computational-Cost Method to Fuse IKONOS Images Using the Spectral Response Function of Its Sensors
}

\author{
María González-Audícana, Xavier Otazu, Octavi Fors, and Jesús Alvarez-Mozos
}

\begin{abstract}
Probably the most popular image fusion method is that based on the intensity-hue-saturation (IHS) transform. Although the spatial enhancement of the IHS-merged images is high, the distortion of its spectral information may also be important. In recent years, several methods have been developed to minimize this problem, being those based on wavelets widely used. However, the high computational cost of these approaches makes them unattractive to applications that involve fast merging of very large volumes of data. In this paper, we present a low computationalcost image fusion method based on the fast IHS transform, which uses the information of the spectral response functions of the lowresolution multispectral (LRM) and high-resolution panchromatic (HRP) sensors to minimize the spectral distortion problem. Using this information, we directly obtain from the HRP image the intensity image that the LRM sensor would observe if it worked at a spatial resolution similar to that of the HRP image. The experimental results carried out on IKONOS images demonstrate that the proposed approach can perform as well as wavelet-based approaches with a lower computational cost.
\end{abstract}

Index Terms-Fast intensity-hue-saturation (IHS) transform, IKONOS images, image fusion, spectral response functions (SRFs).

\section{INTRODUCTION}

W ITH the launch of the IKONOS and QuickBird satellites, the development of new image fusion methods has become an interesting topic for both researchers and users of remote sensing data. Given the design constraints of these sensors, there is an inverse relation between their spatial and spectral resolution. The low-resolution multispectral (LRM) sensor, characterized by capturing the radiance from the different land covers in a high number of bands of the electromagnetic spectrum, does not have an optimal spatial resolution. However, the high-resolution panchromatic (HRP) sensor achieves a high spatial resolution by capturing at the same time the radiance from the land covers in a unique and wider band of the spectrum.

Manuscript received April 26, 2005; revised August 24, 2005. The work of $\mathrm{X}$. Otazu was supported by a grant from the Ramon y Cajal Programme, funded by the Spanish Ministry of Science and Technology.

M. González-Audícana and J. Álvarez-Mozos are with the Department of Projects and Rural Engineering, Public University of Navarre, Campus Arrosadía, 31006 Pamplona, Spain (e-mail: maria.audicana@unavarra.es).

$\mathrm{X}$. Otazu is with the Centre de Visió per Computador, Universitat Autónoma de Barcelona, 08193 Barcelona, Spain (e-mail: xotazu@cvc.uab.es).

O. Fors is with Departament d'Astronomia i Meteorologia, Universitat de Barcelona, E-08028 Barcelona, Spain and also with the Observatori Fabra, 08035 Barcelona, Spain (e-mail: ofors@am.ub.es).

Digital Object Identifier 10.1109/TGRS.2005.863299
An increasing number of applications, such as feature detection, change monitoring, urban analysis, and land cover classification, often demand the use of images with high spatial and spectral resolution for the best achievement of their objectives.

The fusion of LRM and HRP images, with complementary spectral and spatial characteristics, has become a powerful solution providing a single high-resolution multispectral image (HRM) with high spatial and spectral resolution simultaneously.

Probably the most popular image fusion method is the one based on the intensity-hue-saturation (IHS) transformation proposed by Haydn et al. [1]. Although this method provides a HRM image with enhanced spatial quality, its spectral information differs from that of the LRM image.

This modification of the spectral information during the fusion process is not acceptable when the resulting HRM image is going to be used, for example, to extract thematic information through a spectral classification procedure.

Trying to minimize the above-mentioned limitation, Chavez proposed a new fusion method: the high-pass filtering (HPF) method [2], [3]. This method could be considered the predecessor of an extended group of image fusion procedures based on the same principle: extracting from the HRP image the spatial detail information not present in the LRM image, to later inject it into the latter.

In the past few years, several researchers have proposed different image fusion methods based on this concept, employing the discrete wavelet transform [4]-[9], Laplacian pyramid algorithms [10], [11], or "á trous" wavelet transforms [12], [13] to perform the detail extraction and injection processes.

However, no explicit physical information of the detection system has been taken into account in the former fusion methods. Recently, we [14] proposed a new approach that incorporates information from the spectral response of the HRP and LRM sensors to estimate the amount of spatial detail of the HRP image that has to be injected into the $i$ th band of the LRM sensor. This approach, called the window spectral response (WiSpeR), was successfully applied to wavelet-based image fusion methods.

Although the wavelet-based image fusion methods provide HRM images with both high spatial and spectral quality, their high computational cost makes them less attractive when very large volumes of data have to be merged quickly.

In this paper we present a low computational-cost image fusion method, based on the WiSpeR approach, that uses a fast IHS (FIHS) linear transform [15] to inject the spatial information of the HRP image into the LRM image. 
We have determined its validity to fuse IKONOS images by analyzing the spectral and spatial quality of the resulting fused images. Furthermore, we have compared these results with those obtained applying the FIHS fusion method with spectral adjustment proposed by Tu et al. [16], as well as with those obtained applying a FIHS wavelet-based image fusion method.

When applying any of these image fusion methods, it is necessary that the HRP and LRM images be accurately superimposed. Therefore, both images have to be coregistered and the LRM images need to be resampled to make their pixel size the same as that of the HRP image. In this work, we have used a bicubic interpolator to resample the LRM images.

In order to assess the quality of the resulting images, they should be compared with the image that the LRM sensor would theoretically collect if it offered the same spatial resolution as the HRP [17]. Since these images do not exist, we worked with spatially degraded images. The method we have followed to degrade the spatial resolution of the HRM and LRM images is similar to that proposed by Wald et al. [17], i.e., blurring using a low-pass filter and subsampling.

\section{FAST IHS IMAGE FUSION METHOD}

As previously mentioned, the IHS transform is widely used for image fusion purposes. Probably, the widespread use of this transform to merge images relies on its ability to separate the spectral information of an RGB composition in its $H$ and $S$ components, isolating most of the spatial information in the $I$ component [18].

When IHS-based fusion methods are applied, the $I$ image corresponding to the LRM image in IHS space is replaced by a gray-level image with higher resolution $\left(I^{\prime}\right)$ and transformed back into the original RGB space.

Several different algorithms have been developed for converting RGB values into values of IHS [19], [20]. These differ not only in their processing time but also in the methodology used to calculate the value of the $I$ component.

If the conversion model between RGB and IHS spaces used to fuse the LRM and HRP images is linear, the transformations between these spaces are done using the following equations:

$$
\begin{aligned}
& {\left[\begin{array}{c}
I \\
v 1 \\
v 2
\end{array}\right]=\left[\begin{array}{ccc}
1 / 3 & 1 / 3 & 1 / 3 \\
-\sqrt{2} / 6 & -\sqrt{2} / 6 & 2 \sqrt{2} / 6 \\
1 / \sqrt{2} & -1 / \sqrt{2} & 0
\end{array}\right] \cdot\left[\begin{array}{c}
R \\
G \\
B
\end{array}\right]} \\
& {\left[\begin{array}{l}
R^{\prime} \\
G^{\prime} \\
B^{\prime}
\end{array}\right]=\left[\begin{array}{ccc}
1 & -1 / \sqrt{2} & 1 / \sqrt{2} \\
1 & -1 / \sqrt{2} & -1 / \sqrt{2} \\
1 & \sqrt{2} & 0
\end{array}\right] \cdot\left[\begin{array}{c}
I^{\prime} \\
v 1 \\
v 2
\end{array}\right]}
\end{aligned}
$$

where $R, G$, and $B$ represent the corresponding bands of the resized LRM image, $v 1$ and $v 2$ represent the $x$ and $y$ axes in the opponent (R-G,B-Y) Cartesian coordinate system, and $I$ indicates the $z$ axis. $R^{\prime}, G^{\prime}$, and $B^{\prime}$ represent the corresponding bands of the resulting HRM image. The $H$ and $S$ components can be estimated using the following equations:

$$
\begin{aligned}
H & =\tan ^{-1}\left(\frac{v 2}{v 1}\right) \\
S & =\sqrt{v 1^{2}+v 2^{2}} .
\end{aligned}
$$

The implementation of the IHS method following (1) and (2) requires several multiplicative and additive operations, being its application costly from a computational point of view. Tu et al. [15] have proposed a FIHS method to perform the fusion process with lower computational cost, rewritten (2) as

$$
\begin{aligned}
{\left[\begin{array}{l}
R^{\prime} \\
G^{\prime} \\
B^{\prime}
\end{array}\right]=} & {\left[\begin{array}{ccc}
1 & -1 / \sqrt{2} & 1 / \sqrt{2} \\
1 & -1 / \sqrt{2} & -1 / \sqrt{2} \\
1 & \sqrt{2} & 0
\end{array}\right] \cdot\left[\begin{array}{c}
I+\left(I^{\prime}-I\right) \\
v 1 \\
v 2
\end{array}\right] } \\
= & {\left[\begin{array}{ccc}
1 & -1 / \sqrt{2} & 1 / \sqrt{2} \\
1 & -1 / \sqrt{2} & -1 / \sqrt{2} \\
1 & \sqrt{2} & 0
\end{array}\right] \cdot\left[\begin{array}{c}
I+\delta \\
v 1 \\
v 2
\end{array}\right]=\left[\begin{array}{c}
R+\delta \\
G+\delta \\
B+\delta
\end{array}\right] } \\
= & {\left[\begin{array}{l}
R+\delta \\
G+\delta \\
B+\delta
\end{array}\right] }
\end{aligned}
$$

where $\delta=I^{\prime}-I$, being $I$ the mean of $R, G$, and $B$.

If after the fusion process, an RGB-IHS transform is applied to the resulting HRM image, the $H$ component remains unchanged, being the change in the Saturation component directly related with the value of $\delta$ [15]

$$
\frac{S}{S^{\prime}}=\frac{I}{I^{\prime}}=\frac{I}{(I+\delta)}
$$

where $S^{\prime}$ represents the saturation value after fusion.

The higher the value of $\delta$, the higher the change in the $S$ component and the higher the difference between the spectral information of the resulting HRM image and the spectral information of the LRM image.

Traditionally, the HRP image directly replaces the $I$ image, and

$$
\delta=I^{\prime}-I=\mathrm{HRP}-I
$$

When this image fusion approach is used, the spectral information of the resulting HRM image differs from the spectral information of the LRM image, being this difference higher in the green-vegetated areas.

In order to solve this problem, and taking into account that the spectral interval of the HRP sensor covers the spectral intervals of the B, G, R, and near infrared (NIR) bands or the LRM sensor, it is reasonable to suppose that also the NIR band has to be included into the definition of the $I$ component.

This way, and motivated by (5), the IHS transform can be extended from three to four bands by

$$
\left[\begin{array}{c}
B^{\prime} \\
G^{\prime} \\
R^{\prime} \\
\mathrm{NIR}^{\prime}
\end{array}\right]=\left[\begin{array}{c}
B+\delta \\
G+\delta \\
R+\delta \\
\mathrm{NIR}+\delta
\end{array}\right]
$$

where $\delta=I^{\prime}-I=\mathrm{HRP}-I$ and $I=(B+G+R+\mathrm{NIR}) / 4$.

When this image fusion method is applied, the difference between the HRP and the $I$ image is lower from a radiometric point of view, being also lower the change in the $S$ component, and consequently, the distortion of the spectral information of the resulting HRM image.

Hereafter we will name this method extended fast IHS approach (eFIHS). 


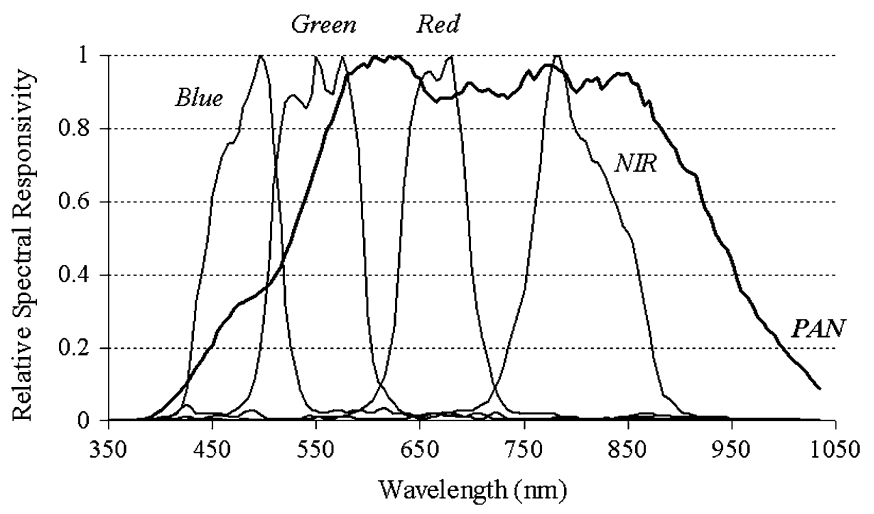

Fig. 1. Spectral Response Functions corresponding to IKONOS sensors.

\section{InTROdUCtion OF THE SPECTRAL RESPONSE FunCTIONS ON THE FAST GIHS IMAGE FUSION APPROACH}

The spectral response function (SRF) of a sensor defines the probability a photon of a given frequency is detected by this sensor. Let $\phi(\nu)$ be the SRF of the HRP sensor and $\varphi_{i}(\nu)$ be the SRF of the $i$-th band of the LRM sensor.

Let the event mi be the detection of a photon by the LRM sensor. The probability of the event $m_{i}$ is

$$
P\left(m_{i}\right)=\int \varphi_{i}(\nu) d \nu .
$$

Similarly, the probability a photon is detected by the HRP sensor can be defined by the probability of the event $p$

$$
P(p)=\int \phi(\nu) d \nu .
$$

In geometrical terms, the probability of the events $m_{i}$ and $p$ can be understood as the area below their corresponding SRFs.

For IKONOS sensors (Fig. 1), the area below $\phi(\nu)$ function is much higher than the area below $\varphi_{i}(\nu)$ function. This means that for a specific observed object, the HRP sensor detects more photons than each $\mathrm{LRM}_{i}$ sensor. For this reason it is important to consider the SRF of both the HRP and $\mathrm{LRM}_{i}$ sensor with the aim of estimating the number of photons that the $\mathrm{LRM}_{i}$ sensor would detect if it worked at a spatial resolution similar to that of the HRP sensor.

Given the event $p$, the probability of the event $m_{i}$ is

$$
P\left(m_{i} \mid p\right)=\frac{P\left(m_{i} \cap p\right)}{P(p)}
$$

being

$$
P\left(m_{i} \cap p\right)=\int \min \left(\varphi_{i}, \phi\right) d \nu
$$

That is, given a photon detected by the HRP sensor, the probability that it is also detected by the LRM sensor is defined by the quotient between the intersection areas of $\varphi_{i}(\nu)$ and $\varphi_{i}(\nu)$ (see Fig. 1), and the area of the function. Similarly, given a photon detected by the LRM sensor, the probability to be detected by the HRP sensor is

$$
P\left(p \mid m_{i}\right)=\frac{P\left(m_{i} \cap p\right)}{P\left(m_{i}\right)}
$$

Note that (11) defines the fraction of HRP photons below the $\operatorname{LRM} \varphi_{i}(\nu)$ function. Similarly, (13) defines the fraction of $\mathrm{LRM}_{i}$ photons below the HRP function.

Let $n_{i}$ and $n_{p}$ be the number of photons detected by the LRM $i$ and the HRP sensors. The total number of photons simultaneously detected by the $\mathrm{LRM}_{i}$ and the HRP sensors $\left(n_{p, i}\right)$ could be defined by

$$
n_{p, i}=P\left(m_{i} \mid p\right) \cdot n_{p}
$$

or by

$$
n_{p, i}=P\left(p \mid m_{i}\right) \cdot n_{i}
$$

If we combine these equations we can predict, given the number $n_{p}$ of photons detected by the HRP sensor, the number $n_{i}^{\prime}$ of photons that the $\mathrm{LRM}_{i}$ sensor should detect [14]

$$
n_{i}^{\prime}=\frac{P\left(m_{i} \mid p\right)}{P\left(p \mid m_{i}\right)} \cdot n_{p} .
$$

\section{FASt IHS Image Fusion ApProACH That TAKES INTO ACCOUNT THE SRF OF THE SENSORS}

Considering that the image fusion method that we propose is based on the FIHS transform extended to four bands, we define the $I$ image as

$$
\begin{aligned}
I & =\sum_{i=1}^{4} n_{i} / 4 \\
I^{\prime} & =\sum_{i=1}^{4} n_{i}^{\prime} / 4=\sum_{i=1}^{4} \frac{P\left(m_{i} \mid p\right)}{P\left(p \mid m_{i}\right)} \cdot \frac{1}{4} \cdot n_{p} .
\end{aligned}
$$

Since we know from data the number of photons detected by the HRP sensor, we can estimate the $I^{\prime}$ image that the LRM sensor would observe if it worked at a spatial resolution similar to that of the HRP sensor.

The difference between the $I$ image obtained from the LRM image and the $I^{\prime}$ image obtained from the HRP image, mainly corresponds to the spatial detail of the second image that is missing in the first one.

The first part of (18) just depends on the SRF of the HRP and LRM sensors, being independent of the images to be fused

$$
\begin{aligned}
\gamma & =\sum_{i=1}^{4} \frac{P\left(m_{i} \mid p\right)}{P\left(p \mid m_{i}\right)} . \\
I^{\prime} & =\frac{1}{4} \cdot \gamma \cdot n_{p} .
\end{aligned}
$$

Taking into account the areas defined by the $\phi(\nu)$ and $\varphi_{i}(\nu)$ functions corresponding to the HRP and LRM sensors, it is possible to estimate the $\gamma$ value for these images fusion. This value is near 0.80 for the IKONOS satellite sensors.

In order to preserve, as much as possible the relative values between the LRM bands, i.e., to preserve the spectral signatures of the observed objects, the added spatial detail is proportional to their original values. 

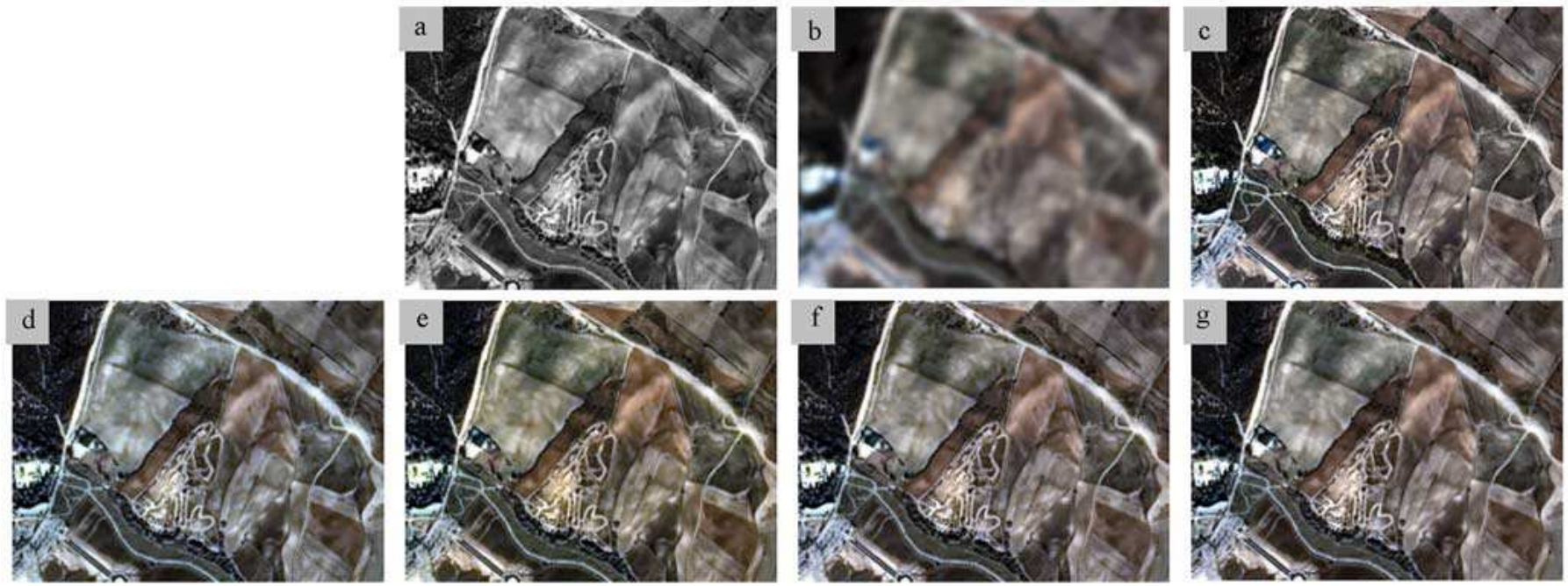

Fig. 2. (a) Part of the initial IKONOS HRP image covering an agricultural area of Madrid (degraded to $4 \mathrm{~m}$ ). (b) Initial IKONOS LRM image (degraded to $16 \mathrm{~m}$ and resampled to $4 \mathrm{~m}$ ). (c) Original IKONOS LRM image (4 m). (d) Fused image using the eFIHS method proposed. (e) Fused image using the method proposed by Tu et al. (2.8 m). (f) Fused image using the eFIHS wavelet-based method. (g) Fused image using the eFIHS SRF method proposed in this paper.

The FIHS image fusion method that we proposed can be represented by

$$
\left[\begin{array}{c}
n_{B}^{\prime} \\
n_{G}^{\prime} \\
n_{R}^{\prime} \\
n_{\mathrm{NIR}}^{\prime}
\end{array}\right]=\left[\begin{array}{c}
n_{B}+\left(\delta \cdot n_{B} / \bar{n}\right) \\
n_{G}+\left(\delta \cdot n_{G} / \bar{n}\right) \\
n_{R}+\left(\delta \cdot n_{R} / \bar{n}\right) \\
n_{\mathrm{NIR}}+\left(\delta \cdot n_{\mathrm{NIR}} / \bar{n}\right)
\end{array}\right]
$$

where $n_{B}^{\prime}, n_{G}^{\prime}, n_{R}^{\prime}$, and $n_{\mathrm{NIR}}^{\prime}$ correspond to the radiance values of the merged HRM image, $n_{B}, n_{G}, n_{R}$, and $n_{\mathrm{NIR}}$ correspond to the radiance values of the resampled LRM image

$$
\begin{aligned}
& \bar{n}=\sum_{i=1}^{4} n_{i} / 4=I \\
& \delta=I^{\prime}-I=\frac{1}{4} \cdot\left(\gamma \cdot n_{p}-\sum_{i=1}^{4} n_{i}\right)
\end{aligned}
$$

being $n_{p}$ and $n_{i}$ the radiance value of the HRP and $\mathrm{LRM}_{i}$ images, respectively.

\section{EXPERIMENTAL RESULTS}

Two sets of IKONOS images collected in November 2004 and March 2005 and covering an agricultural area of Madrid and the city of Pamplona, Spain, respectively, were used as HRP and LRM test images (Fig. 2) to evaluate the performance of the proposed image fusion method (eFIHS SRF) and to compare it with the following fusion methods.

— eFIHS, considering that

$$
\delta=I^{\prime}-I=\mathrm{HRP}-\frac{B+G+R+\mathrm{NIR}}{4}
$$

where $B, G, R$, and NIR correspond to the digital number of the resampled LRM image.

- eFIHS with spectral adjustment applied to the intensity image (eFIHS Tu) [16], considering that

$$
\delta=I^{\prime}-I=\mathrm{HRP}-\frac{a \cdot B+b \cdot G+R+\mathrm{NIR}}{3}
$$

where $a$ and $b$ are weighting parameters defined to take into account that the SRF of the HRP image does not cover the SRF of the blue and green band of the LRM sensor. The value of these parameters was estimated experimentally after the fusion of several IKONOS images. According to the experimental results obtained by Tu et al. [16], the best values for $a$ and $b$ are 0.25 and 0.75 , respectively.

- Extended fast IHS wavelet-based method (eFIHSW), using the "á trous" algorithm to perform the multiresolution wavelet decomposition of the HRP image, considering that

$$
\delta=I^{\prime}-I=\left(I+w_{\mathrm{HRP}}^{\prime}+w_{\mathrm{HRP}}^{\prime \prime}\right)-I=w_{\mathrm{HRP}}^{\prime}+w_{\mathrm{HRP}}^{\prime \prime}
$$

being $w_{\mathrm{HRP}}^{\prime}, w_{\mathrm{HRP}}^{\prime \prime}$ the first and second wavelet coefficients of the HRP image, that collect the spatial detail information of the HRP image corresponding to those structures with a size between the spatial resolution of the HPR image and the spatial resolution of the LRM image. For the practical implementation of the "á trous" algorithm, a two-dimensional filter associated to a scaling function is used. In this work, we use a scaling function that has a B-cubic spline profile.

In order to assess the quality of the merged images, we work with spatially degraded images. The LRM and HRP images were degraded to 16 and $4 \mathrm{~m}$, respectively.

Merged images obtained by different methods have a spatial resolution of $4 \mathrm{~m}$, so the goodness of each image fusion method can be evaluated by comparing the resulting merged images with the IKONOS original one.

This comparison is based on spectral and spatial criteria and is done both visually and quantitatively.

In addition to the quality assessment carried out over the resulting merged images, the computational complexity of each image fusion method was analyzed. 


\section{A. Spectral Quality of the Merged Images}

- The spectral quality of the merged images will be evaluated by comparing their spectral information to that of the original IKONOS LRM image [6], [17]. This comparison is performed quantitatively using the following parameters.

- Correlation coefficient (CC) between the original and the merged images. It should be as close to 1 as possible.

- The Erreur Relative Globale Adimensionnelle de Synthèse (ERGAS) index or relative adimensional global error in the fusion [21]

$$
\text { ERGAS }=100 \frac{h}{l} \sqrt{\frac{1}{n_{c}} \sum_{i=1}^{n_{c}}\left(\operatorname{RMSE}^{2}\left(n_{i}\right) /\left(\bar{n}_{i}\right)^{2}\right)}
$$

where $h$ is the resolution of the HRP image and 1 the resolution of the LRM, $n_{c}$ the number spectral bands considered in the fusion, and $\bar{n}_{i}$ the mean radiance of each spectral band involved in the fusion. The $\operatorname{RMSE}\left(n_{i}\right)$ of each merged band is defined as

$$
\operatorname{RMSE}\left(n_{i}\right)=\sqrt{\sum_{j=1}^{\mathrm{NP}}\left(O_{j}-F_{j}\right)^{2}}
$$

where NP is the total number of pixels in the original and fused image, $O_{j}$ is the radiance value of pixel $j$ in the $i$ th band of the original image, and $F_{j}$ is the radiance value of the pixel $j$ in the $i$ th band of the fused image.

The lower the value of the ERGAS index, the higher the spectral quality of the merged image.

In Table I(A) and (B), we show the values of these indexes resulting from the comparison of the merged images and the original IKONOS LRM images.

In order to quantify the effect that the fusion has over the initial multispectral images ( $16 \mathrm{~m} /$ pixel, spatially degraded image), we show in the first columns the values of the different parameters obtained when these degraded images were compared with the original LRM images ( $4 \mathrm{~m} / \mathrm{pixel})$. Therefore, these first columns reflect the situation before the fusion, while the last columns reflect the situation that ideally should be reached after the fusion.

Higher CC or lower ERGAS values than those showed in the first columns indicate that the analyzed image fusion procedure allows a high-quality transformation of the multispectral content when increasing the spatial resolution.

It can be observed that the fusion method that results in images of the least spectral quality is the one based on the standard FIHS transform.

The image fusion method proposed by Tu et al. allows obtaining HRM images with better spectral quality that the former because the overlap between the SRF of the HRP and LRM sensors is taken into account in the definition of the $I$ component. Even though the ERGAS values are lower after the fusion than before, the color of both the Madrid and the Pamplona merged images [Fig. 2(e) and 3(e)] is slightly greenish and differs from that of the HRM image that would ideally be reached after the fusion, i.e., the original IKONOS multispectral image [Fig. 2(c) and $3(\mathrm{c})]$.
TABLE I

VALUe of THE DifFERENT PARAMETERs ANALYZED to ESTIMATE THE SPECTRAL QUALITY OF THE MERGED IMAGES.

\begin{tabular}{|c|c|c|c|c|c|c|c|}
\hline $\mathbf{A}$ & & Initial & eFIHS & $\begin{array}{c}\text { eFIHS } \\
\text { Tu }\end{array}$ & $\begin{array}{c}\text { eFIHSW } \\
\text { à trous }\end{array}$ & $\begin{array}{c}\text { eFIHS } \\
\text { SRF }\end{array}$ & Ideal \\
\hline \multirow{4}{*}{$\begin{array}{l}\text { Spectral } \\
\text { correlation } \\
\text { coefficient } \\
(C C)\end{array}$} & $\mathrm{X} 1$ & 0.8543 & 0.9336 & 0.9304 & 0.9478 & 0.9459 & 1 \\
\hline & $\mathrm{X} 2$ & 0.8550 & 0.9601 & 0.9694 & 0.9757 & 0.9702 & 1 \\
\hline & X3 & 0.8573 & 0.9591 & 0.9705 & 0.9734 & 0.9685 & 1 \\
\hline & $\mathrm{X} 4$ & 0.8144 & 0.9445 & 0.9566 & 0.9640 & 0.9583 & 1 \\
\hline$\overline{C C}$ & & 0.8452 & 0.9493 & 0.9567 & 0.9652 & 0.9607 & 1 \\
\hline \multicolumn{2}{|l|}{ ERGAS } & 4.422 & 2.747 & 2.331 & 2.149 & 2.215 & 0 \\
\hline \multicolumn{2}{|l|}{ B } & Initial & eFIHS & $\begin{array}{c}\text { eFIHS } \\
\text { Tu }\end{array}$ & $\begin{array}{c}\text { eFIHSW } \\
\text { à trous }\end{array}$ & $\begin{array}{c}\text { eFIHS } \\
\text { SRF }\end{array}$ & Ideal \\
\hline \multirow{4}{*}{$\begin{array}{l}\text { Spectral } \\
\text { correlation } \\
\text { coefficient } \\
(C C)\end{array}$} & $\mathrm{X} 1$ & 0.8832 & 0.9242 & 0.9329 & 0.9550 & 0.9435 & 1 \\
\hline & $\mathrm{X} 2$ & 0.8707 & 0.9560 & 0.9678 & 0.9728 & 0.9701 & 1 \\
\hline & $\mathrm{X} 3$ & 0.8626 & 0.9563 & 0.9708 & 0.9747 & 0.9724 & 1 \\
\hline & $\mathrm{X} 4$ & 0.8351 & 0.9525 & 0.9621 & 0.9648 & 0.9617 & 1 \\
\hline \multicolumn{2}{|l|}{$\overline{C C}$} & 0.8629 & 0.9472 & 0.9584 & 0.9668 & 0.9619 & 1 \\
\hline \multicolumn{2}{|l|}{ ERGAS } & 5.433 & 3.306 & 3.145 & 2.827 & 2.935 & 0 \\
\hline
\end{tabular}
A: IMAGE OF MADRID. B: IMAGE OF PAMPLONA

This color distortion effect does not appear in the merged images obtained when applying the eFIHS SRF fusion method proposed in this work. In this case, the information of the HRP and LRM sensors has been used to spectrally adjust the HRP image and obtain the $I^{\prime}$ image, equivalent to the image that the LRM sensor would acquire if it works at a spatial resolution similar to that of the HRP. The values of the parameters needed to synthesize this $I^{\prime}$ image have been directly obtained from the SRF of the HRP and LRM sensors, instead of having them estimated experimentally. The difference between this $I^{\prime}$ image and the $I$ image obtained from the LRM image corresponds to spatial detail information. A simple subtraction operation between these images $\left(I^{\prime}-I\right)$ allows extracting from the HRP image information concerning to spatial detail only, without attached spectral information.

The concept of the proposed fusion method is similar to the basic concept of the wavelet-based image fusion methods, that is, to extract from the HRP image the spatial detail that is missing in the LRM one, to later inject it into the latter. Doing it this way, the spectral quality of the merged eFIHS SRF images [Figs. 2(g) and 3(g)] is quite similar, both visually and quantitatively, to those obtained using wavelet-based image fusion methods [Figs. 2(f) and 3(f)].

\section{B. Spatial Quality of the Merged Images}

A good fusion method must allow the addition of a high degree of the spatial detail of the HRP image to the LRM image, simultaneously preserving the spectral information of the LRM image.

The spatial quality of the merged images will be evaluated by comparing their information to that of the HRP image. This comparison is performed visually and quantitatively using the same parameters used to assess the spectral quality of the merged images. 

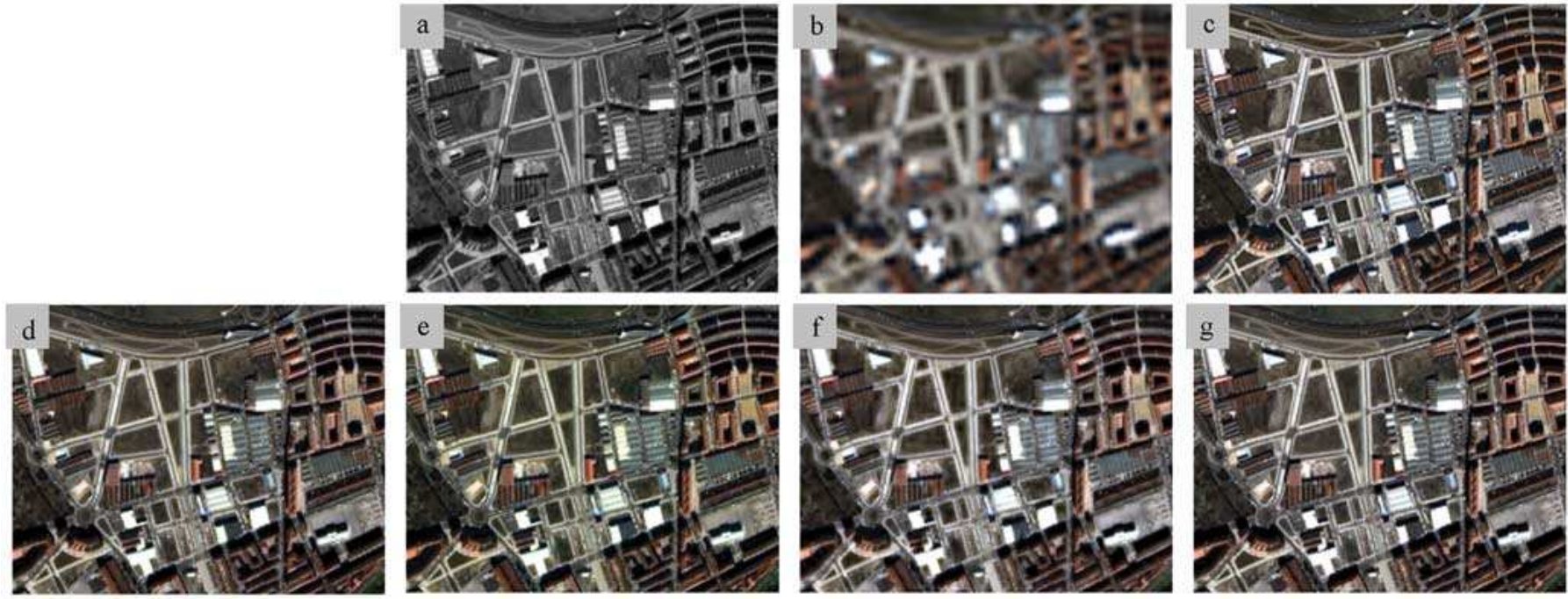

Fig. 3. (a) Part of the initial IKONOS HRP image covering the city of Pamplona (degraded to $4 \mathrm{~m}$ ). (b) Initial IKONOS LRM image (degraded to $16 \mathrm{~m}$ and resampled to $4 \mathrm{~m}$ ). (c) Original IKONOS LRM image $(4 \mathrm{~m}$ ). (d) Fused image using the eFIHS method proposed. (e) Fused image using the method proposed by Tu et al. (2.8 m). (f) Fused image using the eFIHS wavelet-based method. (g) Fused image using the eFIHS SRF method proposed in this paper.

TABLE II

Value of the Different Parameters Analyzed to Estimate the Spatial Quality of the Merged Images. A: IMAGE OF MADRID. B: IMAGE OF PAMPLONA

\begin{tabular}{|c|c|c|c|c|c|c|c|}
\hline A & & Initial & eFIHS & $\begin{array}{c}\text { eFIHS } \\
\text { Tu }\end{array}$ & $\begin{array}{l}\text { eFIHSW } \\
\text { à trous }\end{array}$ & $\begin{array}{c}\text { eFIHS } \\
\text { SRF }\end{array}$ & Ideal \\
\hline \multirow{4}{*}{$\begin{array}{l}\text { Spatial } \\
\text { correlation } \\
\text { coefficient } \\
(\mathrm{sCC})\end{array}$} & $\mathrm{X} 1$ & 0.8195 & 0.9751 & 0.9537 & 0.9557 & 0.9829 & 1 \\
\hline & $\mathrm{X} 2$ & 0.8479 & 0.9936 & 0.9889 & 0.9800 & 0.9940 & 1 \\
\hline & $\mathrm{X} 3$ & 0.8382 & 0.9819 & 0.9735 & 0.9670 & 0.9778 & 1 \\
\hline & $\mathrm{X} 4$ & 0.8387 & 0.9543 & 0.9692 & 0.9708 & 0.9832 & 1 \\
\hline$\overline{s C C}$ & & 0.8360 & 0.9762 & 0.9713 & 0.9683 & 0.9844 & 1 \\
\hline ERGASs & & 4.508 & 1.691 & 1.857 & 1.951 & 1.365 & 0 \\
\hline
\end{tabular}

\begin{tabular}{lccccccc}
\hline \hline B & & Initial & eFIHS & $\begin{array}{c}\text { eFIHS } \\
\text { Tu }\end{array}$ & $\begin{array}{c}\text { eFIHSW } \\
\text { à trous }\end{array}$ & $\begin{array}{c}\text { eFIHS } \\
\text { SRF }\end{array}$ & Ideal \\
\hline Spatial & $\mathrm{X} 1$ & 0.7575 & 0.9670 & 0.9381 & 0.9382 & 0.9731 & 1 \\
correlation & $\mathrm{X} 2$ & 0.7927 & 0.9751 & 0.9587 & 0.9405 & 0.9789 & 1 \\
coefficient & $\mathrm{X} 3$ & 0.7980 & 0.9686 & 0.9515 & 0.9591 & 0.9621 & 1 \\
(sCC) & $\mathrm{X} 4$ & 0.7625 & 0.9085 & 0.9068 & 0.9190 & 0.9454 & 1 \\
\hline$\overline{S C C}$ & & 0.7777 & 0.9548 & 0.9388 & 0.9392 & 0.9649 & 1 \\
\hline ERGASs & 5.782 & 2.955 & 3.059 & 3.268 & 2.296 & 0 \\
\hline \hline
\end{tabular}

In order to minimize the difference in the radiometric values between the HRP image and each band of the merged images, these are modified to match the histogram of the HRP image [22]. This histogram matching is done biasing and stretching the histogram of each merged image to make its mean and standard deviation equal to that of the HRP one.

Higher spatial CC (sCC) or lower spatial ERGAS (ERGASs) values than those shown in the first columns of Table II(A) and (B) indicate that most spatial detail information of the HRP images has been incorporated into the LRM ones during the fusion process.

The addition of this spatial detail is evident in all the merged images when these are visually compared to the initial LRM.
Although there are no perceptible differences between the spatial quality of the merged images when these are compared visually (Figs. 2 and 3), the values of the sCC as well as the ERGASs are better for the merged images obtained applying the eFIHS SRF method proposed in this paper. When this image fusion method is used, the spatial detail information of the HRP image missing in the LRM image is directly extracted, not being necessary to apply any filtering process to the HRP image.

The superior performance of the eFIHS SRF method from a spatial point of view is evident when the difference images between the HRP and each merged band, with its histogram matched to that of the HRP image, are visually compared. Fig. 4 shows the difference images corresponding to the green band of each merged HRM image, biased for display purposes. In the right part of the figure, we include the histogram of each merged image. The narrower the histogram, the higher the similarity between the HRP and the merged image.

Obviously, the radiometric information of both images is different and the difference image will never be a zero-mean and zero-standard deviation image.

Although the numerical results are acceptable for the eFIHSW image, the visual analysis of the difference image [Fig. 4(d)] reveals that the amount of spatial detail of the HRP image injected into the LRM one is oversized in some cases (dark gray lines or edges) and undersized in others (light gray lines or edges). This effect can also be noticed in the eFIHS and the eFIHS Tu images, but with a much lower incidence. A simple look to Fig. 4(e) reveals that there are no spatial detail differences between the HRP and the eFIHS SRF images. Consequently, it is possible to affirm that the whole detail information of the HRP image has been incorporated into the fused image when the eFIHS SRF method was used.

Taking into account that we have assessed both the spectral and the spatial quality of the merged images using the same index, it is possible to estimate the global quality of these images by the average of the ERGAS and ERGASs values (Table III). 

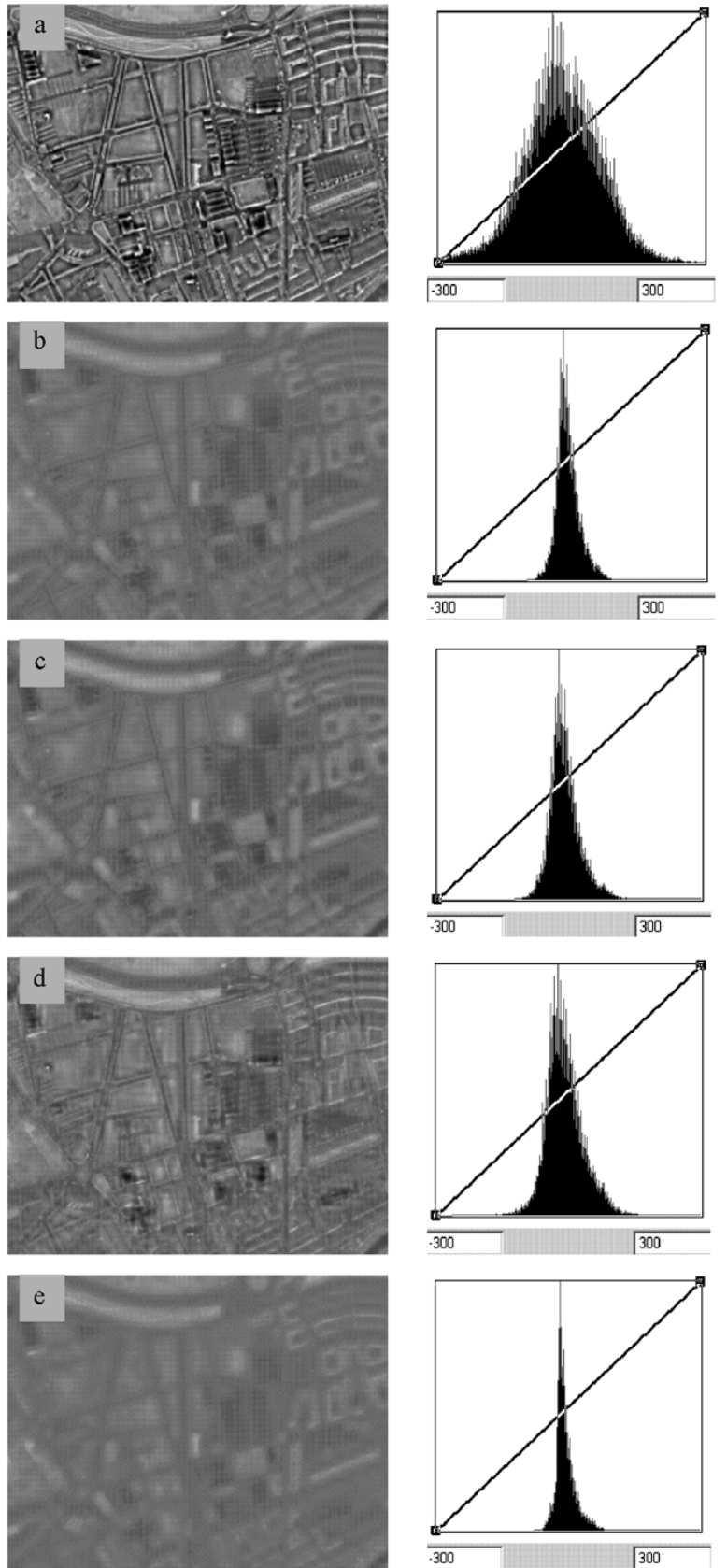

Fig. 4. (a) Part of the difference image between the HRP and the GREEN band of the initial image covering the city of Pamplona (degraded to $16 \mathrm{~m}$ and resampled to $4 \mathrm{~m}$ ). (b) Difference image between the HRP and the GREEN band of the fused eFIHS image. (c) Difference image between the HRP and the GREEN band of the fused eFIHS Tu image. (d) Difference image between the HRP and the GREEN band of the fused eFIHW image. (e) Difference image between the HRP and the GREEN band of the fused eFIHS SRF image.

The eFIHS SRF method proposed in this work provides HRM images with higher global quality than the eFIHSW à trous method, being lower than its computational cost, as will be demonstrated in Section V-C.

\section{Computational Cost of the Different IHS-Based Image Fusion Methods}

Here, the computational complexity of the proposed eFIHS SRF fusion algorithm is discussed and compared to that of the
TABLE III

AVERAge ERGAS VALue For the DifFERENT MERged IMAgeS. A: IMAGE OF MADRID. B: IMAGE OF PAMPLONA

\begin{tabular}{lcccccc}
\hline \hline A & Initial & eFIHS & $\begin{array}{c}\text { eFIHS } \\
\text { Tu }\end{array}$ & $\begin{array}{c}\text { eFIHSW } \\
\text { à trous }\end{array}$ & $\begin{array}{c}\text { eFIHS } \\
\text { SRF }\end{array}$ & Ideal \\
\hline Average ERGAS & 4.465 & 2.219 & 2.089 & 2.050 & 1.790 & 0 \\
\hline \hline & & & & & & \\
\hline \hline & Initial & eFIHS & $\begin{array}{c}\text { eFIHS } \\
\text { Tu }\end{array}$ & $\begin{array}{c}\text { eFIHSW } \\
\text { à trous }\end{array}$ & $\begin{array}{c}\text { eFIHS } \\
\text { SRF }\end{array}$ & Ideal \\
\hline Average ERGAS & 5.607 & 3.130 & 3.102 & 3.047 & 2.615 & 0 \\
\hline \hline
\end{tabular}

other IHS-based fusion approaches used in this work to fuse IKONOS images (a spatial resolution ratio of $4: 1$ is considered since it is a common ratio on usual image fusion tasks).

In order to carry out this analysis, we will compute the number of operations involved in each algorithm. Considering that the difference between these algorithms lies in the way the $\delta$ image is calculated, we will focus this analysis just on this part of the image fusion algorithms.

Let $N$ be the number of columns and rows of the images to be merged and $F \times F$ the size of the filter used to obtain the wavelet coefficients when the eFIHSW à trous method is applied.

According to (24), the number of operations needed to obtain the $\delta$ image when the eFIHS method is used is

$$
\mathrm{eFIHS}_{\mathrm{ops}}=5 \cdot N^{2} \text {. }
$$

According to (25), the number of operations needed to obtain the $\delta$ image when the eFIHS Tu method is used is

$$
\text { eFIHS } \mathrm{Tu}_{\mathrm{ops}}=7 \cdot N^{2} \text {. }
$$

When the eFIHSW method is applied, the following differences are computed to obtain the wavelet coefficient images $w_{\mathrm{HRP}}^{\prime}, w_{\mathrm{HRP}}^{\prime \prime}$

$$
\begin{aligned}
w_{\mathrm{HRP}}^{\prime} & =\mathrm{HRP}-\mathrm{HRP}_{\mathrm{FIL}(L 1)} \\
\mathrm{eFIHS} \mathrm{Tu}_{\mathrm{ops}} & =7 \cdot N^{2}
\end{aligned}
$$

where $\mathrm{HRP}_{\mathrm{FIL}(L 1)}$ is the HRP image filtered with an $F \times F$ convolution mask, and $\mathrm{HRP}_{\mathrm{FILL2}}$ is the $\mathrm{HRP}_{\mathrm{FIL}(L 1)}$ filtered with the same convolution mask. The value of each pixel on this $\mathrm{HRP}_{\mathrm{FIL}(L 2)}$ image is calculated applying the convolution mask coefficients over just one out of two neighboring pixels, both in the row and column directions. The number of operations required to obtain the $\delta$ image in this case is

$$
\mathrm{eFIHSW}_{\mathrm{ops}}=\left(3+2 F^{2}\right) \cdot N^{2} \text {. }
$$

As mentioned before, we have used a B-cubic spline function to perform the "á trous" wavelet decomposition. This function leads to a convolution mask of $5 \times 5$, consequently being $F=5$.

All the fusion methods mentioned above are directly applied to digital counts (DC) images. To apply the image fusion algorithm proposed in this work (eFIHS SRF) it is necessary to convert DC to radiance values (HRP to $n_{\mathrm{HRP}}, B$ to $n_{B}, G$ to $n_{G}, R$ to $n_{R}$, and NIR to $n_{\mathrm{NIR}}$ ). Obviously, these conversions have a computational cost that has not been considered in this analysis because they are not directly involved in the fusion process. According to (23), the number of operations needed to obtain the $\delta$ 


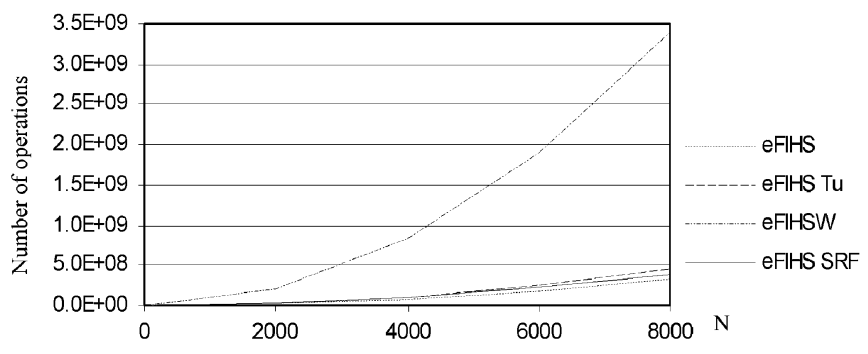

Fig. 5. Relative computational cost of the image fusion algorithms analyzed in this work

image following the eFIHS SRF method proposed in this work is

$$
\text { eFIHS } \mathrm{SRF}_{\mathrm{ops}}=6 \cdot N^{2} \text {. }
$$

Fig. 5 shows how the number of operations required to compute the $\delta$ image increases when the size or the number of pixels $\left(N^{2}\right)$ of the HRP and LRM images to be fused increase.

The eFIHSW method is the most expensive, being the computational cost of the other three methods very similar.

\section{CONCLUSION}

The image fusion approach presented in this work, uses the information in the SRF of the IKONOS HRP and LRM sensors to obtain a fast approximation to the $I^{\prime}$ image that the LRM sensor would acquire if it worked at a spatial resolution similar to that of the HRP sensor. This $I^{\prime}$ image contains the spatial detail information of the HRP image not present in the LRM one. The injection of this spatial detail into the LRM image is done using a fast IHS transform. The HRM images obtained using this method present similar spectral quality and higher spatial quality than the images obtained using the wavelet-based image fusion methods used in this work, being the computational cost of this method lower than that of the used waveletbased method.

\section{ACKNOWLEDGMENT}

The authors would like to thank the European Space Imaging Company for providing the IKONOS multispectral and panchromatic images used in this work. They would also like to thank J. L. Saleta for his useful comments about this paper.
[4] B. Garguet-Duport, J. Girel, J. M. Chassery, and G. Pautou, "The use of multiresolution analysis and wavelet transform for merging SPOT panchromatic and multispectral image data," Photogramm. Eng. Remote Sens., vol. 62, pp. 1057-1066, Sept. 1996.

[5] J. Zhou, D. L. Civco, and J. A. Silander, "A wavelet transform method to merge Lndsat TM and SPOT panchromatic data," Int. J. Remote Sens., vol. 19 , no. 4, pp. 743-757, 1998.

[6] T. Ranchin and L. Wald, "Fusion of high spatial and spectral resolution images: The ARSIS concept and its implementation," Photogramm. Eng. Remote Sens., vol. 66, pp. 49-61, Jan. 2000.

[7] D. A. Yocky, "Image merging and data fusion by means of the discrete two-dimensional wavelet transform," J. Opt. Soc. Amer. A., vol. 12, pp. 1834-1841, Sept. 1995

[8] Y. Zhang, "A new merging method and its spectral and spatial effects," Int. J. Remote Sens., vol. 20, pp. 2003-2014, May 1999.

[9] M. González-Audícana, J. L. Saleta, O. G. Catalán, and R. García, "Fusion of multispectral and panchromatic images using improved IHS and PCA mergers based on wavelet decomposition," IEEE Trans. Geosci. Remote Sens., vol. 42, no. 6, pp. 1291-1299, Jun. 2004.

[10] T. A. Wilson, S. K. Rogers, and M. Kabrisky, "Perceptual-based image fusion for hyperspectral data," IEEE Trans. Geosci. Remote Sens., vol. 35, no. 4, pp. 1007-1017, Jul. 1997.

[11] B. Aiazzi, L. Alparone, S. Baronti, and A. Garzelli, "Context-driven fusion of high spatial and spectral resolution images based on oversampled multiresolution analysis," IEEE Trans. Geosci. Remote Sens., vol. 40, no. 10 , pp. $2300-2312$, Oct. 2002.

[12] J. Nuñez, X. Otazu, O. Fors, A. Prades, V. Palá, and R. Arbiol, "Multiresolution-based image fusion with additive wavelet decomposition," IEEE Trans. Geosci. Remote Sens., vol. 37, no. 3, pp. 1204-1211, May 1999.

[13] Y. Chibani and A. Houacine, "The joint use of IHS transform and redundant wavelet decomposition for fusing multispectral and panchromatic images," Int. J. Remote Sens., vol. 23, pp. 3821-3833, Sep. 2002.

[14] X. Otazu, M. González-Audícana, O. Fors, and J. Nuñez, "Introduction of sensor spectral response into image fusion methods: Application to wavelet-based methods," IEEE Trans. Geosci. Remote Sens., vol. 43, no. 10, pp. 2376-2385, Oct. 2005.

[15] T.-M. Tu, S.-C. Su, H.-C. Shyu, and P. S. Huang, "A new look at IHS-like image fusion methods," Inf. Fusion, vol. 2, pp. 177-186, 2001.

[16] T.-M. Tu, P. S. Huang, C.-L. Hung, and C.-P. Chang, "A fast IntensityHue-Saturation fusion technique with spectral adjustment for IKONOS imagery," IEEE. Geosci. Remote Sens. Lett., vol. 1, no. 4, pp. 309-312, Oct. 2004.

[17] L. Wald, T. Ranchin, and M. Mangolini, "Fusion of satellite images of different spatial resolution: Assessing the quality of resulting images," Photogramm. Eng. Remote Sens., vol. 63, pp. 691-699, Jun. 1997.

[18] C. Pohl and J. L. Van Genderen, "Multisensor image fusion in remote sensing: Concepts, methods and applications," Int. J. Remote Sens., vol. 19, pp. 823-854, Mar. 1998.

[19] A. R. Smith, "Color gamut transform pairs," Comput. Graph., vol. 12 , pp. 12-19, Aug. 1978.

[20] Graphics Standard Planning Committee of ACM/SIGGRAPH, "Status report," Comput. Graph., vol. 13, no. 3, 1979.

[21] L. Wald, "Quality of high resolution synthesized images: Is there a simple criterion?", in Proc. Int. Conf. Fusion of Earth Data, Jan. 2000, pp. $99-105$.

[22] M. Lillo-Saavedra, C. Gonzalo, A. Arquero, and E. Martinez, "Fusion of multispectral and panchromatic satellite sensor imagery based on tailored filtering in the fourier domain," Int. J. Remote Sens., vol. 26, pp. 1263-1268, Mar. 2005

\section{REFERENCES}

[1] R. Haydn, G. W. Dalke, J. Henkel, and J. E. Bare, "Applications of the IHS color transform to the processing of multisensor data and image enhancement," in Proc. Int. Symp. Remote Sensing of Arid and Semi-Arid Lands, Cairo, Egypt, 1982, pp. 559-616.

[2] P. S. Chavez, "Digital merging of Landsat TM and digitized NHAP data for 1:24.000 scale image mapping," Photogramm. Eng. Remote Sens., vol. 52, pp. 1637-1646, Oct. 1986.

[3] P. S. Chavez and J. Bowell, "Comparison of the spectral information content of Landsat thematic mapper and SPOT for three different sites in Phoenix, Arizona region," Photogramm. Eng. Remote Sens., vol. 54 pp. 1699-1708, Dec. 1988.

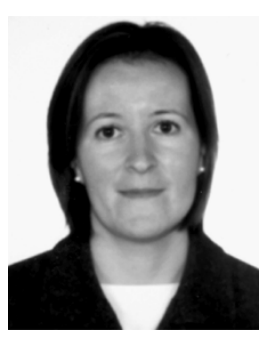

María González-Audícana received the degree in agricultural engineering in 1996 and the Ph.D. degree in agricultural engineering, with a thesis based on image fusion, in 2002, both from the Public University of Navarre, Pamplona, Spain.

Since 1997, she has been an Assistant Professor at the Department of Projects and Rural Engineering, Public University of Navarre, where she is currently involved in research activities on multisensor data fusion, multispectral image segmentation, and image classification for agricultural management purposes. 


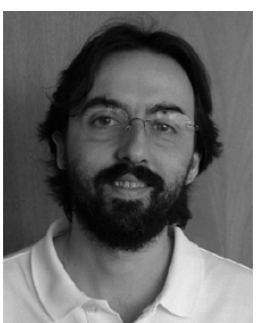

Xavier Otazu received the B.A. degree in physics from the University of Barcelona, Barcelona, Spain, in 1994, the M.S. degree in remote sensing from the Institut d'Estudis Espacials de Catalunya (IEEC), Catalunya, Spain, in 1999, and the Ph.D. degree in physics from the University of Barcelona, in 2001.

Since 1994, he has been working on applications of wavelets to astronomical and remote sensing image and data processing techniques such as image deconvolution, image fusion, period analysis, and data clustering. He is with the R\&D Remote Sensing Group at the Institut Cartográfic de Catalunya, working on SAR image processing, image fusion, and image classification. He is also currently a Ramón y Cajal Researcher at the Computer Vision Center and a Lecturer in the Computer Science Department, Universitat Autonoma de Barcelona, working in color and texture image analysis, especially in the study of the human color visual system and the mathematical description of its perceptual processes using wavelets.

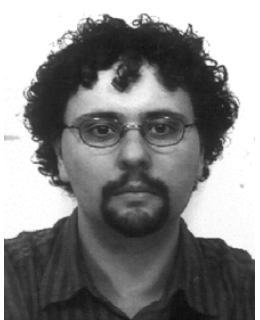

Octavi Fors received the B.A. degree in physics from the University of Barcelona, Barcelona, Spain, in 1996, and the M.S. degree in remote sensing from the Institut d'Estudis Espacials de Catalunya (IEEC), Catalunya, Spain, in 1999. He is currently pursuing the $\mathrm{Ph} . \mathrm{D}$. degree in physics at the University of Barcelona.

Since 1996, he has been working on image processing techniques, both in the astronomy and remote sensing fields. In particular, he has developed a special interest in image deconvolution, image fusion, and multiresolution-based disparity analysis.

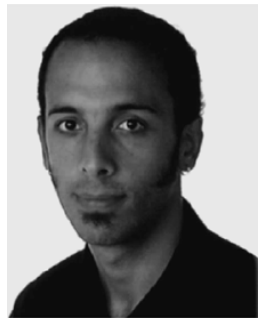

Jesús Álvarez-Mozos received the engineering and Ph.D. degrees in agricultural engineering from the Public University of Navarre, Navarre, Spain, in 2001 and 2006, respectively, with a thesis on radar-based soil moisture retrieval.

Since 2001, he has been an Assistant Professor in the Department of Projects and Rural Engineering, Public University of Navarre. His research interests include remote sensing data processing, especially image fusion and hydrological applications of remote sensing. 